\title{
1 Open Access publishing in Medicine: lights and shadows.
}

2 Nicola Bernabò ${ }^{1}$, Luca Valbonetti ${ }^{2}$, Alessandra Ordinelli ${ }^{2}$, Rosa Ciccarelli ${ }^{1}$, Barbara Barboni ${ }^{1}$.

$3{ }^{1}$ Faculty of Bioscience and Agro-Food and Environmental Technology, University of Teramo, Teramo, Italy;

4• $\quad{ }^{2}$ National Research Council - Institute of Biochemistry and Cell Biology, Rome, Italy

5

6 *Corresponding Author:

7 E-mail: nbernabo@unite.it (NB)

\section{ORCID:}

9 NB: 0000-0001-5140-9656

10 BB: 0000-0003-0231-8066

11

12

13

14 


\section{Abstract}

16 The widespread use of internet has had enormous consequences in changing the way of accessing to scientific literature 17 in all domains of knowledge and, in particular, in medicine. One of the most important related factors is the idea of 18 making research output freely available: the so-called Open Access (OA) option. OA is considered very important in 19 spreading of knowledge, breaking down barriers in benefit of research, and increasing the impact of research outputs 20 within the scientific community.

21 Here, we carried out a comparison between Non-Open Access (NOA) and OA medical Journals in terms of growing 22 rate, geographical distribution, and the impact on scientific community.

23 We collected the bibliometric data on the scientific Journals indexed in Scopus starting from 2001 to 2016 published 24 either as NOA or OA. Then, we analysed the number of Journals, their geolocalization, their impact on the scientific community, and the parameters as SJR, $H$ index, and cites for document (2 years).

26 As a result, we found that while the number of NOA Journals is virtually stable, that of OA is dramatically increasing, 27 with a growing rate higher than $400 \%$ in 2016. Then, the majority of OA Journals are published in developing Countries 28 (Brazil, India, South Africa, South Korea, New Zealand, Serbia, and Poland) and their impact within researchers is 29 lower compared to the NOA Journals.

30 In conclusion, our data provide an updated and unprecedented picture of OA adoption in medical field, with its lights 31 and shadows.

Key Words: Open Access, Medicine, bibliometrics, Scopus, citation advantage.

\section{Article Highlights}

- We are seeing in medicine a huge increase in the number of Journals that use OA;

- This trend is mainly due to the increase in publishing Journals in Countries with emergent economies;

- The use of OA for Journals in the medical field, did not guarantee a vantage in term of bibliometric parameters.

MCS: 92 C99 


\section{Introduction}

Widespread public access to the World Wide Web, possible nowadays with negligible costs for a large number of people (at least in developed Countries), has deeply changed the way of accessing to scientific knowledge, with enormous consequences in the spread of information among scientists and between scientists and the lay public. In this context, starting from the late 90 's, the issue free access to the scientific literature has been raised, the so-called open access $(\mathrm{OA})$, possible available so far only to scientific Journal subscribers.

More precisely, Open access $(\mathrm{OA})$ is defined the access, to research outputs free from all access and many use restrictions (https://opensource.com/resources/what-open-access). It is possible to distinguish different forms of OA. Gratis OA: the online access free of charge, and libre OA, the online access free of charge plus various additional usage rights, usually under the Creative Commons licenses. In addition, there are possible Golden OA or Green OA options. Golden OA is provided by Journals that make the research output immediately available on line and generally requires the payment of a publication fee (from hundreds to thousands of dollar), green OA has no cost and is provided by researchers who self-archive their own research outputs in a public repository (such as institutional and central repositories). There are several important reasons related to the adoption of OA. Firstly, it allows the spreading and sharing of knowledge in developing countries, where research Institutions often find it difficult to pay for subscriptions required to have access to scientific literature. In other words, ideally, all researchers (but also journalists, politicians, civil servants, or interested laypeople) could benefit from OA, being able to dispose of scientific knowledge without barriers or restrictions. Then, several funding Agencies, either at international or national level, explicitly and mandatorily require that researchers publish their research output in OA Journals. For instance, EU in the H2020 program requires that all research outputs be published with OA, with the aim of "building on previous research results (improved quality of results), encouraging collaboration and avoiding the duplication of effort (greater efficiency), speeding up innovation (faster progress to market means faster growth) and involving citizens and society (improved transparency of the scientific process)" ${ }^{1}$. The same policy has been adopted by the Italian Ministry of Educations, University and Research (MIUR) for the research outputs from projects funded by the Projects of Relevant National Interest (PRIN) programme (http://attiministeriali.miur.it/anno-2016/novembre/dd-07112016-(1).aspx). In addition, important Professional Organizations encourage the use of OA such as the International Mathematical Union that in 2001 stated: "Open access to the mathematical literature is an important goal" and encouraged them to "[make] available electronically as much of our own work as feasible" to "[enlarge] the reservoir of freely available primary mathematical material, particularly helping scientists working without adequate library access." (http://cr.yp.to/bib/imucall.html). Again, one of the most important reasons for OA adoption is that it is recognised as an important tool to guarantee free access to scientific literature, considering that taxpayers pay for most of the research through government 
74 grants, and they must have the right to visualize to the results of what they have funded. Finally, it has also been 75 suggested that scientists should benefit from the OA adoption because it could assure an increase in the impact of their 76 research by potentially increasing the number of research papers citations (the so called "citational advantage" of OA) ${ }^{2}$. 77 This last point, in particular, has attracted the attention of researchers because the evaluation of research outputs impact, 78 assessed as number of citations, is a consolidated practice as demonstrated by the popularity of citation-dependent 79 bibliometric indexes (i.e. H index, IF, SJR, ect...) ${ }^{3,4}$. It is often used in contexts related with the funding research 80 policy, with the developing of carriers, as well as with the scientific collaboration policy ${ }^{5}$. Consequently, it has a deep 81 impact on scientists as well as on the public at large. However, the concept of OA citation advantage is still debated and 82 there are conflicting hypotheses on this issue due to the complexity of the matter ${ }^{6,7}$. For instance, there are several 83 interfering factors that could affect the number of citations and are correlated to the OA citations without a causal factor such as the number of co-authors, Authors self-selection of higher quality articles for OA (Quality or Selection bias), the earlier dissemination via preprints/OA repositories (Early access bias) (http://www.istl.org/10-winter/article2.html). In that context, we performed an analysis (based on quantitative bibliometric data) aimed to study the dynamics related to the temporal and geographical spreading of OA publishing and the impact (evaluated in terms of number of documents, number of citations per document, SJR and $\mathrm{H}$ index) of medical scientific literature published under NOA or OA conditions. For this purpose, we accessed the data indexed in Scopus, which is a repository that includes Journals that fulfil basic quality requirements, thus avoiding to consider lower quality publications. We referred our analysis to medicine because the access, spreading, and impact of scientific knowledge in this field could have very important issues either ethical ${ }^{8,9}$ or related to the data quality ${ }^{10}$.

\section{Materials and Methods}

\section{Data collection}

As data source, we selected the Journals indexed on Scimago Journal and Country Rank control of indexed Journals. In fact, to be included in Scopus Journals, it is necessary to meet some eligibility criteria that assure a minimum of quality. In particular, they should consist of peer-reviewed content; they must be published on

102 should be relevant and readable for an international audience, and the Journals should have a publication ethics and 103 publication malpractice statement. Additionally, Journals should have a publication history of at least two years ${ }^{11}$. If a

104 Journal meets all these criteria, after an accurate peer-review process, it is indexed. Thus, a myriad of low quality 
105

106

107

108

109

110

111

112

113

114

115

116

117

118

119

120

121

122

123

124

125

126

127

128

129

130

131

132

133

134

135

Journals are excluded from our analysis. Indeed, mostly in recent years, we are seeing the proliferation of OA Journals with no regulation and scientific quality, often as part of the so called predatory publishing ${ }^{12,13}$. Since in the present study, we were interested in assessing the impact of OA on the real scientific community, we analysed only the Journals present in Scopus (which is at least considered a necessary, but not completely sufficient requisite to be considered reliable). Clearly, this implies a limitation in our analysis, due to the fact that Scopus covers less than half of the biomedical Journals listed in Ulrich's periodical classified as “Academic/Scholarly"14.

We used SCImago because it is a publicly available portal, thus it guarantees free access to data, for proofing or further analyses. In addition, it includes the indicators developed from the information contained in the Scopus database (Elsevier B.V.) related to scientific Journals grouped according to subject area (27 major thematic areas), subject category (313 specific subject categories) or country. Citation data is drawn from over 34,100 titles from more than 5,000 international publishers and country performance metrics from 239 countries worldwide. Importantly, it assures a quality control of the Journals listed (see Discussion section).

We carried out our analysis considering the data referring to the Subject Area (SA): "Medicine"; Subject Category: "All subject categories"; years: 2001, 2006, 2011, 2016.

We obtained a first list of all the Journals (AJ), and then using the option "Only Open Access Journals", we obtained the list of OA Journals. Finally, we computed the list of non OA (NOA) Journals by subtracting the OA Journals from the AJ list.

For each Journal and each year, we listed the following parameters:

- SCImago Journal Rank (SJR): used by SCImago as a measure of scientific influence of scientific Journals. It accounts for both the number of citations received by the Journal and the importance of the Journals such citations come from. It expresses the average number of weighted citations received in the selected year by the documents published in the selected journal in the three previous years, i.e. weighted citations received in year $\mathrm{X}$ to documents published in the journal in years $\mathrm{X}-1, \mathrm{X}-2$ and $\mathrm{X}-3$.

- $\quad H$ index: a researcher with an $h$ index has published $h$ papers, each of which has been cited in other papers at least $h$ times. It is a metric that attempts to measure the impact of a Journal on the scientific community;

- Cites per Document (2 years): defined as the average citations per document in a 2 years period. It is computed considering the number of citations received by a journal in the current year to the documents published in the two previous years, i.e. citations received in year $\mathrm{X}$ to documents published in years $\mathrm{X}-1$ and $\mathrm{X}-2$.

- Country: the Country where the Journal has been published, i.e. where the Publisher has its legal address. This could lead to some imprecision, since it is possible that the location of publishers office is not related to the Journal area of interest. Consequently, this issue could in part bias the discussion of this specific point. 
137 For an extensive explanation of the parameters used, see https://www.scimagojr.com/help.php.

\section{Geolocalization}

139 Data related to the selected papers were processed for geospatial analysis by $\mathrm{Sci}^{2} \mathrm{Tool}\left(\mathrm{Sci}^{2} \mathrm{Team}\right)$. We generated a

140 visualization of the choropleth map that shows the geographic distribution of the NOA and OA Journals distinguished

141 by shades of colour for each Country, proportional to the number of Journals published.

\section{Data analysis}

143 The data were checked for normalcy by using the D'Agostino and Pearson test, which computes the skewness and

144 kurtosis of data under analysis and quantifies how far the distribution is from the Gaussian distribution. As a result, it

145 computes a single $\mathrm{P}$ value from the addition of these discrepancies.

146 Then, we processed the data as normal or not-normal, following the needs (see figure legends for the specific test used

147 in each analysis). All the calculations have been performed with Past3 or Microsoft Excel 2013. The bibliometric data

148 analysis on Subject Categories was carried out when we found a number of records $>5$, to assure a sufficient quality of

149 the statistical analysis. In particular, we compared the data referred to the NOA and OA Journals, in terms of number of

150 Journal, published in 2001, 2006, 2011, and 2016, Country of origin, and bibliometric indexes (SJR, H index, number

151 of citations/Document). In respect to this last analysis, we computed the best curve fitting comparing different models

152 (e.g. linear, exponential, power law, logarithmic, polynomial models) and we assessed the best fitting based on the

153 highest coefficient of determination $\mathrm{R}^{2}$.

154

155 Results

\section{Temporal and geographical trends in NOA and OA publishing in medicine}

157 As shown in Figure 1, it is evident that in comparison to the examined period, the number of NOA Journals increased

158 less than $10 \%$, reaching a plateau of about 5200-5300 Journals. The OA, on the contrary, are strongly increasing (four-

159 time increase in the last 15 years) with a markedly increasing trend.

160 We carried out the Geolocalization of NOA and OA Journals in accord with the period taken in examination. As a

161 result, we computed the number of NOA and OA Journals active in 2001, 2006, 2011 and 2016. Data are listed in

162 Supplementary Material 2 and graphically represented in Figures 2A-D. 


\section{Could OA publishing assure a higher impact of research output in medical field?}

165 As impact parameters, we evaluated SJR, H index and the number of Cites/Documents, referred to the years 2001,

1662006,2011 and 2016.

167 As it is evident in Figures 3, 4 and 5, all these parameters follow an exponential distribution, with a negative exponent

168 ranging between 2 and 3.

169 Interestingly, the exponents of SJR and Cites/Document are always lower in OA when compared with NOA, while the

170 time trend seems to be stable. On the contrary, $\mathrm{H}$ index was higher in OA in 2001, then it gradually decreased until

171 2016, when it was higher than that of NOA Journals, as displayed in Figure 6.

172 The differences in term of bibliometric indexes are summarized in Table 1.

173 As summarized in Supporting Material 1, we analysed all bibliometric parameters studied in all Subject Categories,

174 with a minimum number of 5 Journals. On a total of 465 parameters, we found that in about $82 \%$ of cases (381/465)

175 there aren't differences. While in $18 \%$ (84/465) we found statistically significant differences ( $\mathrm{p}<0.05)$. In all cases, the

176 impact parameters referred to NOA were higher than those of OA Journals were.

177

\section{Discussion}

179 Here, for the first time to our knowledge, we carried out an analysis aimed to take an updated picture of OA adoption in

180 publishing in Journals in the medical field using Scopus as data source (https://www.scopus.com/). In particular, we

181 evaluated the number of NOA and OA Journals in medicine over the time (2001-2016). Evidently, the number of NOA

182 Journals increased less than $10 \%$ in the last 15 years and seems to have reached a plateau. On the contrary, the OA

183 Journals in 2001 were 310 (vs. 4580 NOA Journals) while in 2016 they were 1320, with an increase of over 400\%,

184 furthermore the trend seems to be continuously increasing with an exponential trend $\left(y=191.6 e^{0.504} ; \mathrm{R}^{2}=0.976\right)$.

185 Although these data are only indicative, it is possible to affirm that the OA model seems to be a very successful, so that

186 if the trend does not change in the future it could be possible that in less than 15 years, the number of OA Journals will

187 overcome that of NOA Journals. See Figure 1. Consequently, we analysed the data related to the most productive

188 Countries, responsible for the increasing number of OA products. In this regard, it is very interesting to note that the

189 Countries mostly active in the increasing of number OA Journals are Brazil, India, South Africa, New Zealand, Poland,

190 Serbia, and South Korea, while the Countries with a leader position and a well-defined scientific tradition (USA, UK)

191 did not display a comparable trend. From this datum, we can infer that OA could open new markets to the publishing

192 system. Interestingly, three of them are BRICS (Brazil, Russia, India, China and South Africa) members. They are large 
193 and fast-growing national economies, characterized by specific demographic and geographic situations that play a

194 specific and leading role with a significant influence on regional and global affairs. In 2015, they represented 3.6 billion

195 people, $22 \%$ of the world economy (gross world product) and nearly $60 \%$ of its growth ${ }^{18}$. Brazil today is the leading

196 Country in Latin America, with more than 1,700 journals alone on the Open Journal System. In 2015, 36\% of the

197 Brazilian research output was open access (compared to $12-14 \%$ for France, Germany, US or UK) ${ }^{19,20}$. The most

198 important OA platform in Brazil is the Scientific Electronic Library On-line, SciELO (http://www.scielo.br/). It is "an

199 electronic library covering a selected collection of Brazilian scientific journals" (http://www.scielo.br/), and it is integral

200 part of a project developed by - Fundação de Amparo à Pesquisa do Estado de São Paulo (FAPESP), in partnership

201 with the Latin American and Caribbean Center on Health Sciences Information (BIREME), supported by - Conselho

202 Nacional de Desenvolvimento Científico e Tecnológico (CNPq). The main aim of the project is "the development of a

203 common methodology for the preparation, storage, dissemination and evaluation of scientific literature in electronic

204 format" (http://www.scielo.br/). This has had the great advantage to make available, without costs, the research results

205 to the researchers and lay people, mainly in Latin America. But, unfortunately, as criticized, without considering the

206 quality of Journals indexed ${ }^{18}$. To date (February 2018) it accounts more than 20,000 documents (82.6\% research

207 articles), mainly of Brazilian Authors (70.9\%), of which more than half are in English (56.8\%), with particular regard to

208 Health Sciences (38.7\%), Human Sciences (17.8\%), and Agricultural Sciences (14.1\%)(all the information related

209 SCIelo is listed in Supporting Material 2).

210 In India, the OA policy is supported by important research organisations, laboratories and universities (10).

211 Consequently, more than half of Indian Journals are characterized by OA publishing and most of them do not charge

212 author fees ${ }^{21}$. This, in one hand increased the availability and the visibility of Indian research product; on the other

213 hand, it makes the way to the spread of predatory publishing. As reported by Beall (https://beallslist.weebly.com/)

214 several Journals declare to be headquartered in the United States, United Kingdom, Canada or Australia, but are really

215 located in Pakistan, Nigeria or, mainly, India.

216 South Africa has the second largest economy in Africa, after Nigeria, and it is Country more industrialized in Africa. It

217 accounts for $35^{\%}$ of Africa gross domestic product and is ranked as an upper-middle-income economy by the World

218 Bank, and is the only African member of the G-20 group. Because of its technological infrastructures it has quickly

219 developed online journals, repositories, and a variety of other tools and platforms based on OA, South Africa is the

220 leading African Country in terms of OA policies ${ }^{22}$. To date, the international Directory of Open Access

221 Journals records 79 open access journals produced in South Africa (http://roar.eprints.org/).

222 In South Korea the OA adoption is growing very rapidly in recent years and its improvement is part of the great effort

223 in sustaining education and high technology research ${ }^{23}$. 
224

226

228

230

New Zealand Institutions are quickly moving towards adoption OA policies and mandates. For instance, contracts for the Marsden Fund, New Zealand's fundamental research fund, include a clause mandating that Researchers must share their data, meta-data and samples within 12 months of completion of the project (https://aoasg.org.au/new-zealandopen-access-journals/).

The specific situation of OA publishing of Poland and Serbia, the only two European Countries examined, are exhaustively described in two monographs to which we refer the reader ${ }^{24,25}$.

Then, we assessed three different parameters (SJR, H index and number of cites/document) that are widely used to evaluate the impact of Journals, related to citations. In particular, SJR assigns different values to citations depending on the importance of the journals and where they come from. Consequently, citations coming from important Journals, will be considered (weighted) more than those from less important Journals. The SJR calculation is similar to the Eigenfactor score in the Web of Science database ${ }^{15} . H$ index, suggested in 2005 by Jorge E. Hirsch, which represents the measure of impact and productivity of an Author, a group of Authors or a Journal, as well as the number of cites/document, related to the unweighted number of citations.

We are conscious that the use of citation-related indexes could be criticized for several valid reasons and that the relationship between the number of citations of a scientific paper and the importance of its content is not always obvious, and the values of the parameters used, are strongly dependent on the database accessed and on the specific discipline. For these reasons, we limited their use as indicators of Journal impact rather than Journal quality ${ }^{16,17}$.

The comparison of bibliometric parameters between NOA and OA Journals demonstrates that they are often statistically different trough the examined years, with NOA Journals showing higher values.

More interesting, we compared them in the different subject categories (SC) of medical areas. Here too, we found several important differences, with the parameters of NOA Journals higher than those of OA ones in about $20 \%$ of the cases (85 of 464).

Another interesting thing is the study of frequency distribution of these indexes. Indeed, in agreement with the Bedford's law, they are distributed following a power law, with a negative exponent (usually near 3). As it is evident from Figure 6, for SJR and Cites/Document parameters the exponent of NOA Journals is virtually always higher (lower in absolute value), with a stable trend over the time. It means that that the distribution of values is flatter. In other words, a higher number of Journals (in proportion) have lower values and a lower number of Journals has higher values. These results seem to be in contradiction with the previous results from Eysenbach that found a "Citation Advantage of Open Access Articles" ${ }^{2}$. Actually, he compared articles published in the same Journal (PNAS), which adopted a hybrid publishing policy (it means that part of articles were published with NOA and part with OA). Adopting a logistic regression model, controlling for potential confounders, he found that OA articles compared to NOA articles remained 
255

256

257

258

259

260

261

262

263

264

265

266

267

268

269

270

271

272

273

274

275

276

277

278

279

280

281

282

283

284

twice as likely to be cited in the first 4-10 months after publication (odds ratio= $2.1[1.5-2.9]$ ), with the odds ratio increasing to 2.9 (1.5-5.5) 10-16 months after publication. Then, it was concluded that "Articles published as an immediate OA article on the journal site have higher impact than self-archived or otherwise openly accessible OA articles." ${ }^{2}$ In other words, it was suggested that the adoption of OA could assure a higher impact on the scientific community, when compared to NOA.

In our opinion, the apparent difference with our results is due to the different rationale of the investigation. Eysenbach compared the citations of articles published in the same Journal, PNAS, which is a very peculiar Journal: it is a general science journal, with an $H$-index $=648$, and an $\operatorname{SJR}=6.321(2016)^{2}$. While this study is focused on PNAS, which is a Journal with very peculiar characteristics, we compared a very large number of Journals referred to whole the medical area, consequently his conclusion are not directly comparable with those from our data analysis.

Anyway, the question remains open, as proven by the conclusion of another very interesting work on PNAS citations of NOA and OA papers from Patrick Gaulé and Nicolas Maystre ${ }^{12}$. In fact, they concluded that "at least part of the large number of citations received by open access papers is due to a self-selection effect rather than a spreading (or causal) effect". In means, that Authors preferentially published as OA the articles they consider of higher quality and the reason of the citation number could actually be the quality itself. As a proof, they found that OA articles receive a higher number of cumulative citations also in articles published in Nature, Science, or Cell, that are authored by scientists performing cutting-edge science and that can hardly be expected to lack access to the NOA scientific literature.

Further, the Open Access Citation Advantage Service (OACA), which is a non-profit, member organisation comprised of a diverse body of academic institutions, library consortia, funding bodies, research institutes and some publishers published on its website (https://sparceurope.org/) a list of 70 articles until 2015, of which 66\% (46) declare a citation advantage for OA papers, $24 \%$ (17) found no advantages and $10 \%$ (7) were inconclusive. Unfortunately, the inclusion criteria of the articles and the quality check methods were not discussed, and then this data cannot be considered actually reliable.

Ultimately, the relevance of OA in sharing research results among scientists and/or lay people is challenged by different ways of sharing. In particular, widely accessed repositories are often used, as for instance ResearchGate (https://www.researchgate.net/), as well as other platforms (SciHub, OpenAccessButton, Unpaywall); the assessment of their impact is out of the scope of this paper, see for reference the comment: "Who's downloading pirated papers? Everyone." by John Bohannon (http://www.sciencemag.org/news/2016/04/whos-downloading-pirated-paperseveryone). 


\section{Conclusions}

286 Despite this study has limitation due to the fact that Scopus only distinguishes between OA and non-OA journals, and 287 not $\mathrm{OA}$ and non-OA articles thus the analysis inevitably is unable to account for articles published OA in hybrid 288 journals.

289 Anyway, we think that our analysis could contribute to the knowledge of the OA model spreading in the medical field, 290 offering a new perspective since it takes into account the Journals listed in Scopus referable to Medicine published in a 291 large window of time (2001-2016) that coincides with the onset and spreading of OA literature. In conclusion, we could 292 affirm that:

293 - We are seeing in medicine a huge increase in the number of Journals that use OA;

294 - This trend is mainly due to the increase in publishing Journals in Countries with emergent economies (Brazil, 295 India, South Africa, New Zealand, Poland, Serbia, and South Korea), rather than in developed occidental economies;

- The use of OA for Journals in the medical field, in the last 15 years did not guarantee a vantage in term of impact, but rather they seem often to be characterized by lower impact parameters.

In 1999, The World Conference on Science held under the auspices of UNESCO and ICSU stated that "Equal access to science is not only a social and ethical requirement for human development, but also essential for realizing the full potential of scientific communities worldwide and for orienting scientific progress towards meeting the needs of humankind" ${ }^{26}$. We could conclude, after almost 20 years, that researchers of the emerging countries significantly adopt a new and rapidly expanding way to share medical knowledge.

\section{References}

310 (1) European Commission, Horizon Europe, Open science Early knowledge and data sharing, and open $311 \quad$ collaboration. 2021.

312 (2) Eysenbach, G. Citation Advantage of Open Access Articles. PLoS Biol. 2006, 4, $692-698$.

313 (3) Doja, A.; Eady, K.; Horsley, T.; Bould, M. D.; Victor, J. C.; Sampson, M. The H- Index in Medical 

in the United States $\square$ : An Epidemiologic Study of Scholastic Production o No t Co. 2017, 395-398.

317 (5) Bornmann, L.; Daniel, H. The State Of. 2009.

318 (6) Kreiner, G. The Slavery of the H-Index — Measuring the Unmeasurable. 2016, 10, 1-4.

319 (7) Costas, R. The H-Index $\square$ : Advantages, Limitations and Its Relation with Other Bibliometric Indicators at the Micro Level. 2007, 1, 193-203.

(8) Parker, M. The Ethics of Open Access Publishing. BMC Med. Ethics 2013, 14, 1.

(9) Grant-kels, J. M. International Journal of Women 's Dermatology The Ethical Arguments in Support of Open Access Journals $\square$. Int. J. Women's Dermatology 2017, 3, 4-5.

(10) Wicherts, J. M.; Bakker, M.; Molenaar, D. Willingness to Share Research Data Is Related to the Strength of the Evidence and the Quality of Reporting of Statistical Results. 2011, 6, 1-7.

(11) Scopus. Scopus Journal FAQs $\square$ : Helping to Improve the Submission \& Success Process for Editors \& Publishers. 1-17.

(12) Seo, J.; Chung, H.; Yun, J.; Park, J. Y.; Park, E.; Shin, E.; Of, D.; Journal, S.; Indicator, R.; Grouse, L.; et al. Getting Cited: Does Open Access Help? Antioxid Redox Signal 2013, 342, 1-4.

(13) Sen, C. K. Commitment to Intellectual Honesty and Personal Responsibility. Antioxid Redox Signal 2013, 39.

(14) Gadella, B. M.; Harrison, R. a P. Capacitation Induces Cyclic Adenosine 3',5'-Monophosphate-Dependent, but Apoptosis-Unrelated, Exposure of Aminophospholipids at the Apical Head Plasma Membrane of Boar Sperm Cells. Biol. Reprod. 2002, 67, 340-350.

(16) Bernabò, N.; Greco, L.; Mattioli, M.; Barboni, B. A Scientometric Analysis of Reproductive Medicine. Scientometrics 2016, 109. 
(18) Schöpfel, J. Introduction $\square$ : Open Access to Scientific Information in Emerging Countries To Cite This

(19) Nassi-calò, L. Challenges for Sustainability of the Open Access Model $\square$ : Brazilian Health Journals. 2016, 0-2.

343 (20) Packer, A. L.; Meneghini, R.; Guedes, R. D. SciELO at 15 Years: Raison d'être, Advances, Challenges and the Future; The Pioneering Vision of the SciELO Founders.; 2014. $110,1-9$.

(23) Shin, E. Scholarly Journal Publishing and Open Access in South Korea. Ser. Rev. 2012, 38, 99-104.

(24) Ševkušić, M.; Janković, Z.; Kužet, A. Open Access Journals in Serbia Policies and Practices. 
bioRxiv preprint doi: https://doi.org/10.1101/2021.11.08.467687; this version posted November 8, 2021. The copyright holder for this preprint (which was not certified by peer review) is the author/funder, who has granted bioRxiv a license to display the preprint in perpetuity. It is made available under aCC-BY-NC-ND 4.0 International license.

361 Table 1: list of $p$ values obtained by comparing NOA and OA Journals (data compared with Mann-Whitney $U$ test)

362

363

\begin{tabular}{cccc} 
Year & SJR & H Index & Cites/Document (2 years) \\
\hline 2001 & $<\mathbf{0 , 0 0 0 1}$ & $\mathbf{0 , 0 0 3 7}$ & $\mathbf{0 , 0 2 0 7}$ \\
2006 & $\mathbf{0 , 0 3 3 3}$ & 0,2052 & 0,9653 \\
2011 & $\mathbf{0 , 0 0 3 7}$ & $<\mathbf{0 , 0 0 0 1}$ & 0,8190 \\
2016 & 0,0733 & $<\mathbf{0 , 0 0 0 1}$ & $<\mathbf{0 , 0 0 0 1}$
\end{tabular}

364

365 Were $p<0.05$ NOA are always higher than OA.

366

367 


\section{Figures}

A

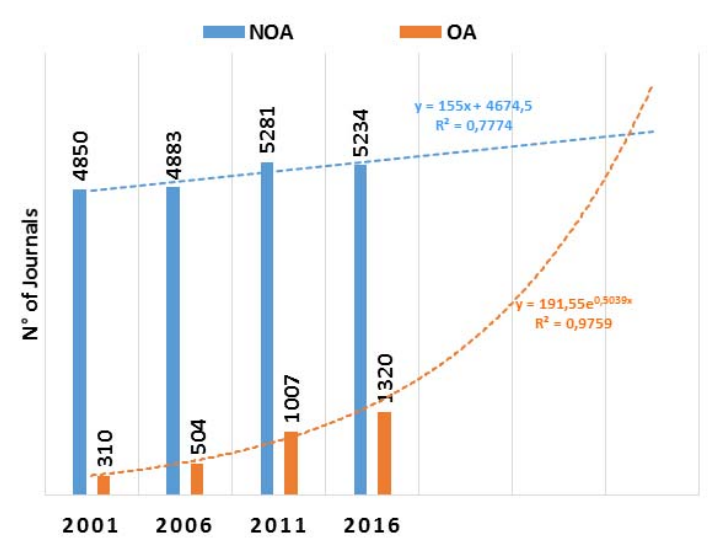

B

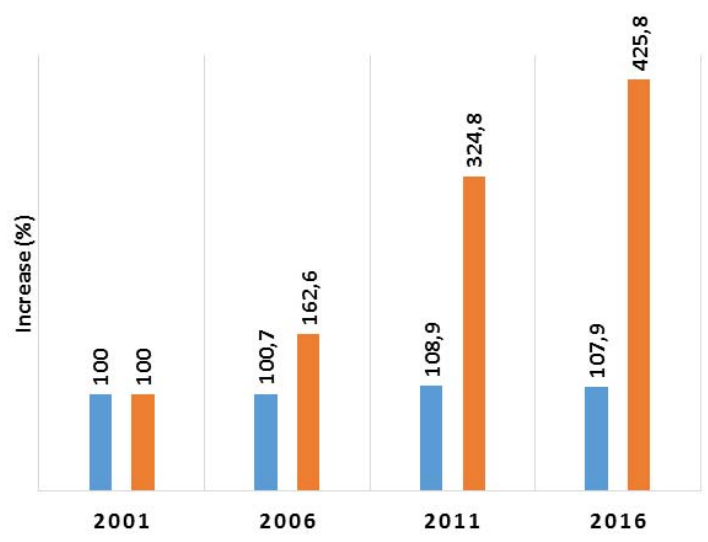

369

370

371

372

373

Figure 1: number of NOA and OA Journals in "Medicine" in 2001, 2006, 2011, and 2016 (Scopus).

Data shown the number of Journals in each year as absolute number (Panel A) and as percentage (the value in $2001=$ $100 \%$ ) (Panel B). The light blue and orange dot lines represent the temporal trend in NOA and OA publishing of NOA and OA Journals, respectively.

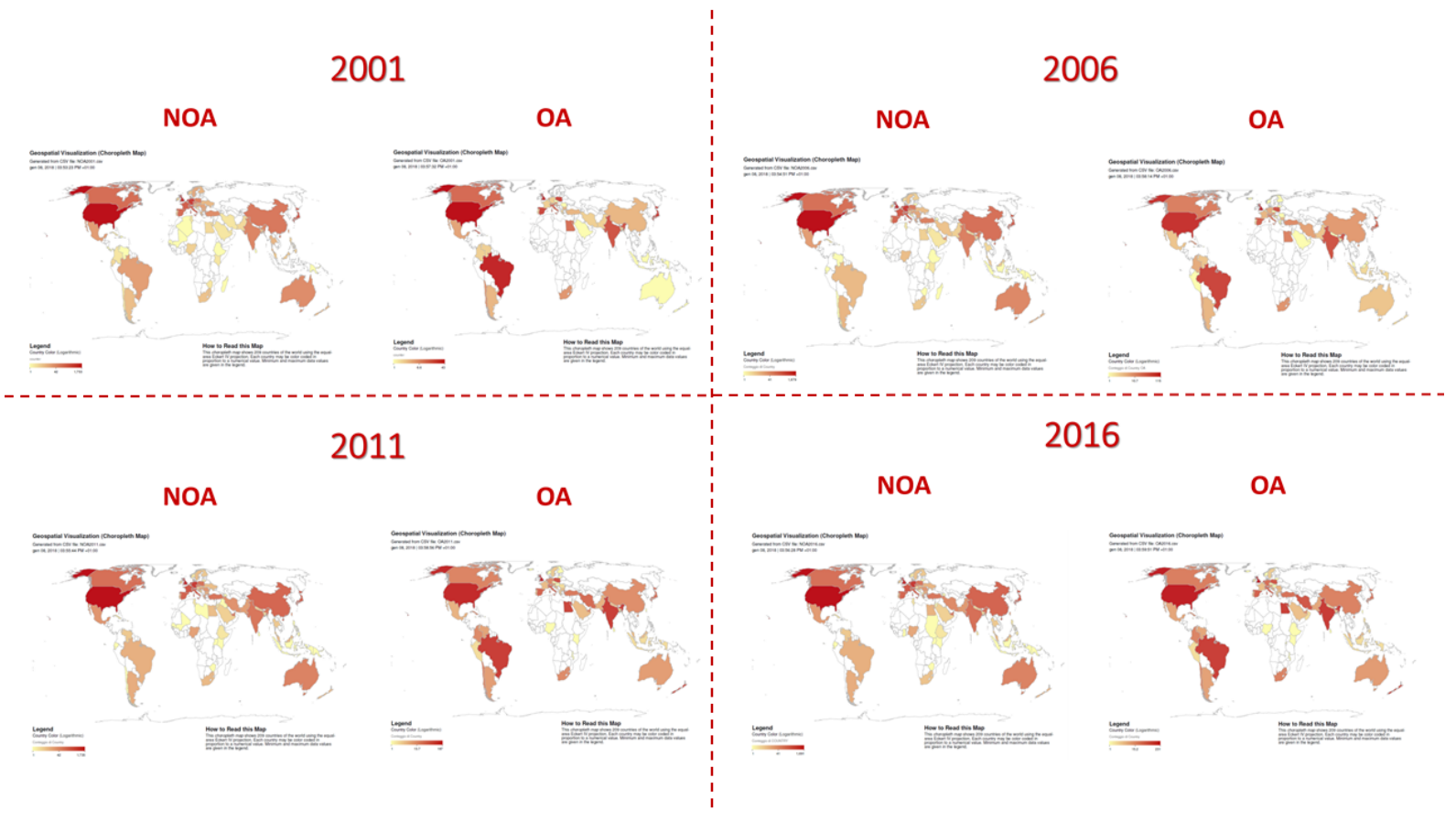

375 Figure 2: choropleth map of NOA and OA Publishers of Journals in "Medicine" in 2001, 2006, 2011, and 2016 376 (Scopus).

377 The reported choropleth map shows the geographic distribution of the NOA and OA Journals differentiated by shades 378 of colour for each Country on the basis of the number of published Journal. 

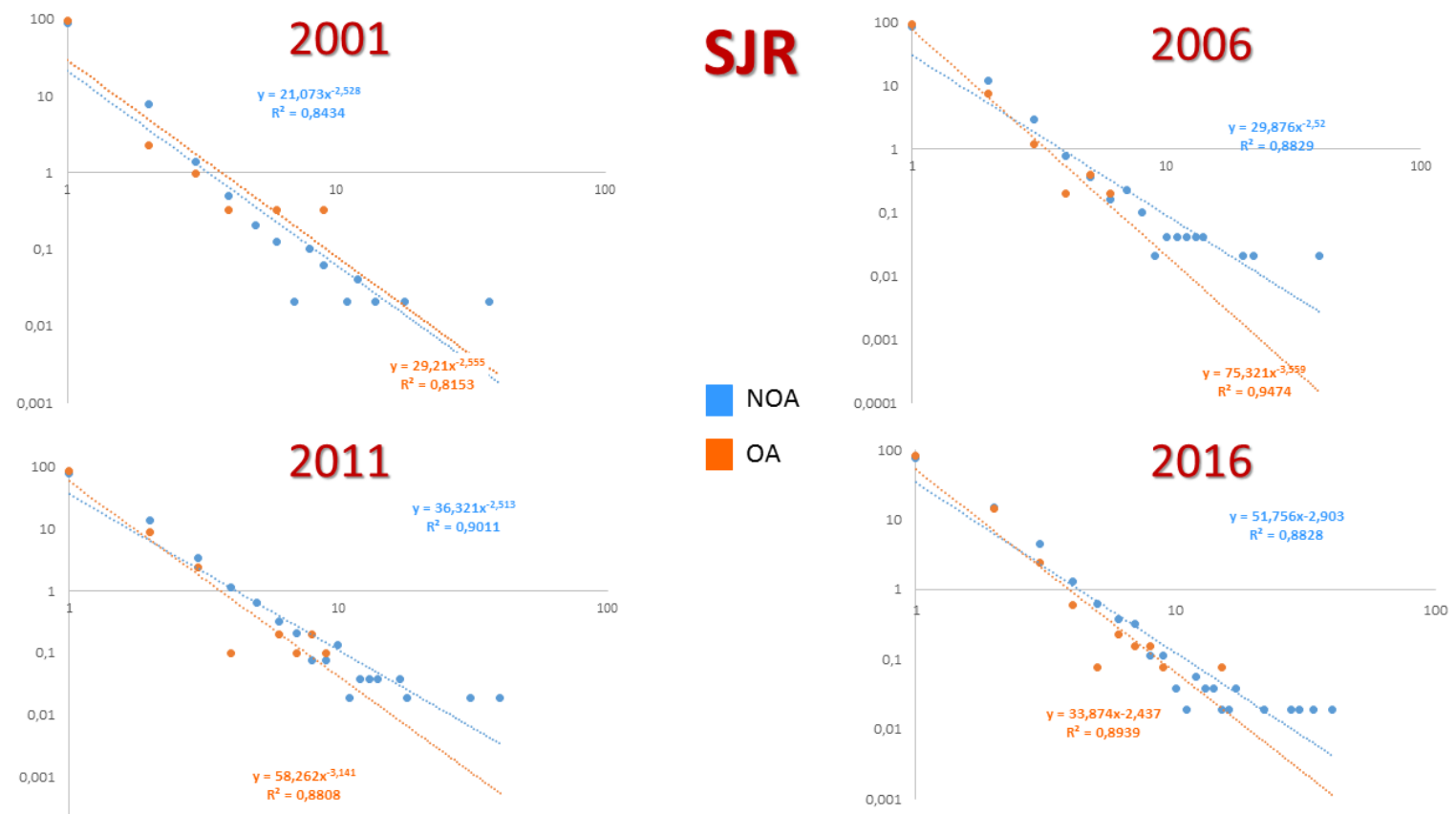

0,0001

Figure 3: graphs showing the distribution of SJR in 2001, 2006, 2011, and 2016 in NOA and OA Journals.

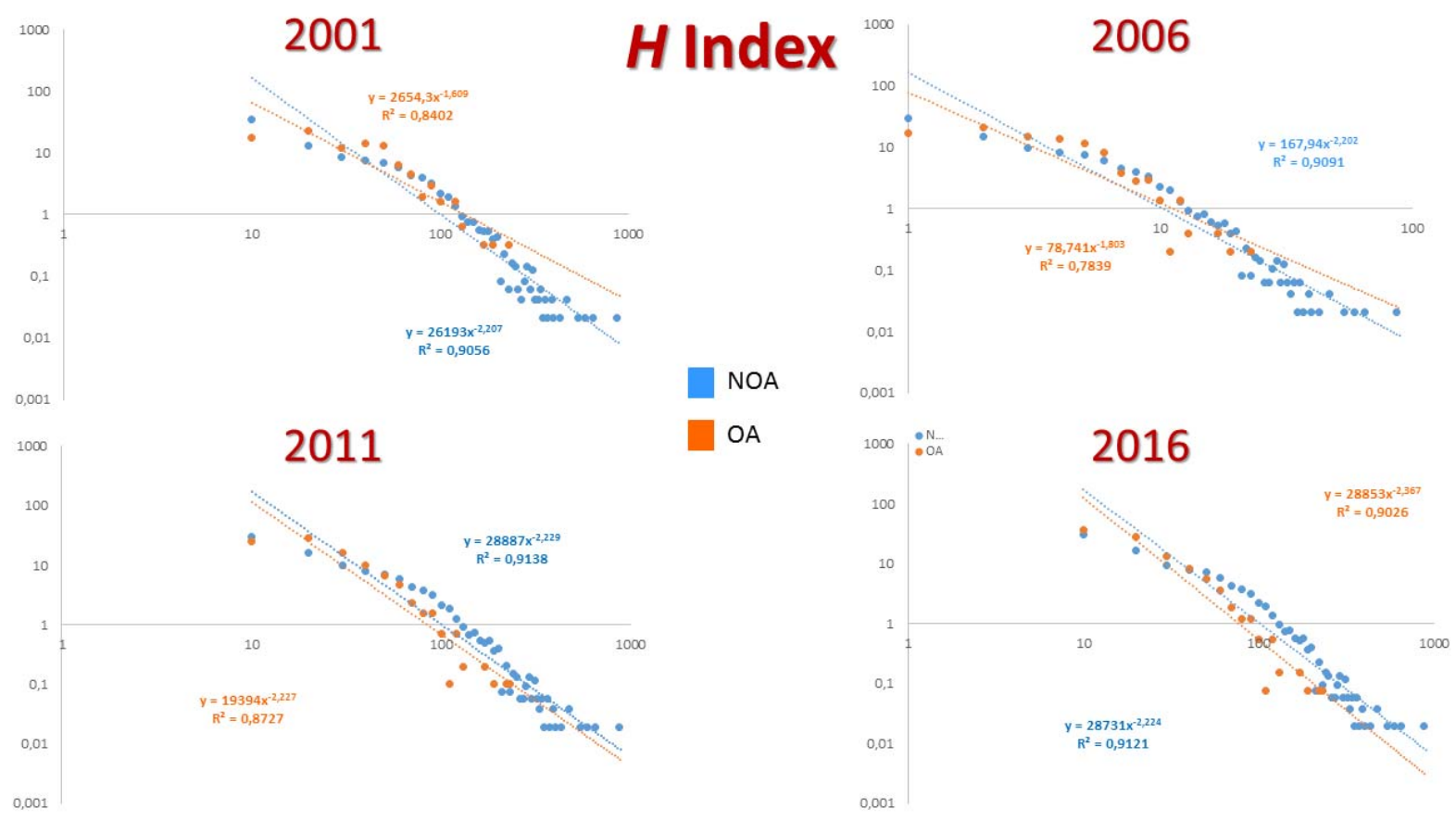

382 Figure 4: graphs showing the distribution of Cites/Document in 2001, 2006, 2011, and 2016 in NOA and OA Journals. 

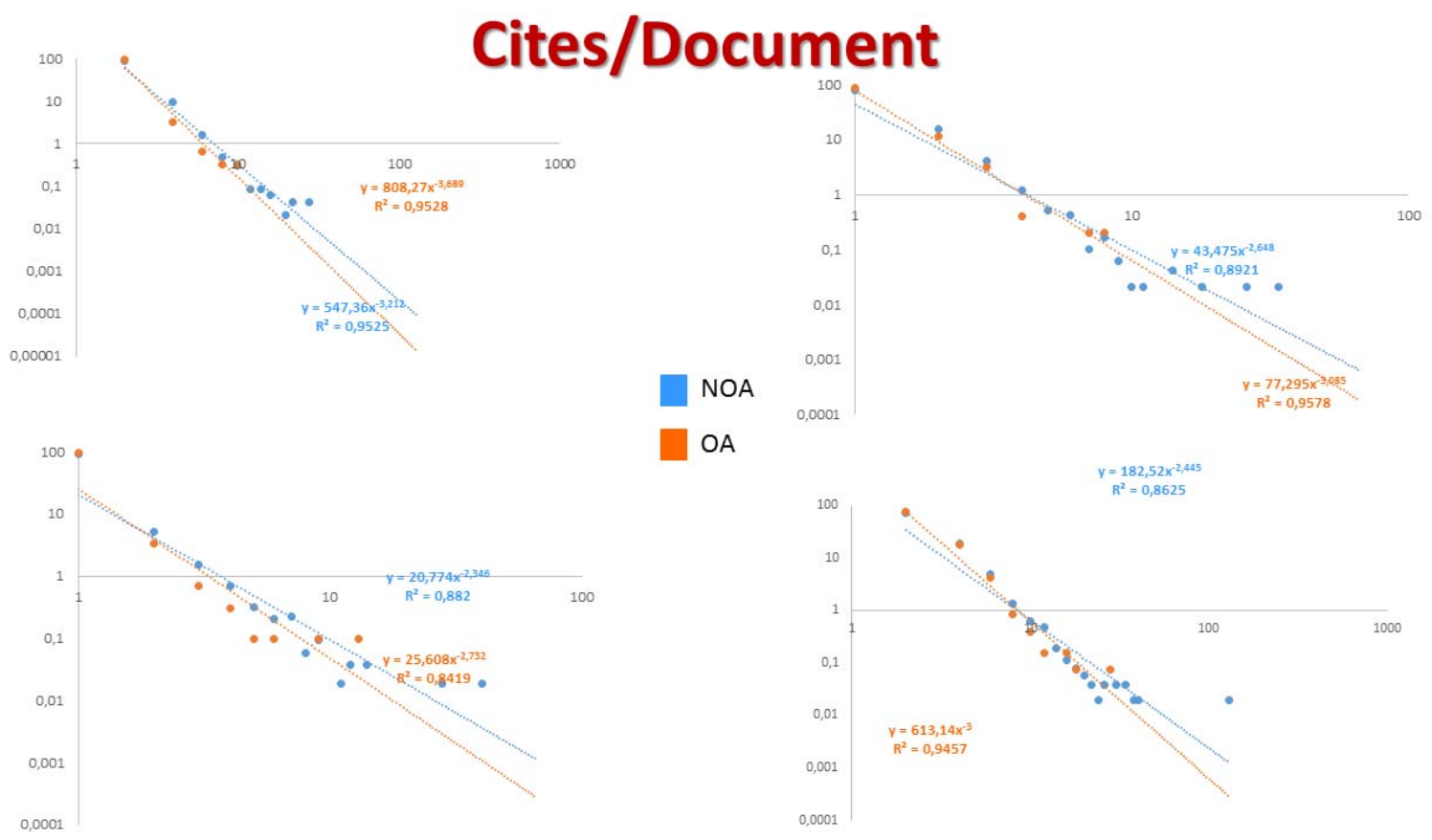

384 Figure 5: graphs showing the distribution of $\mathrm{H}$ index in 2001, 2006, 2011, and 2016 in NOA and OA Journals. 

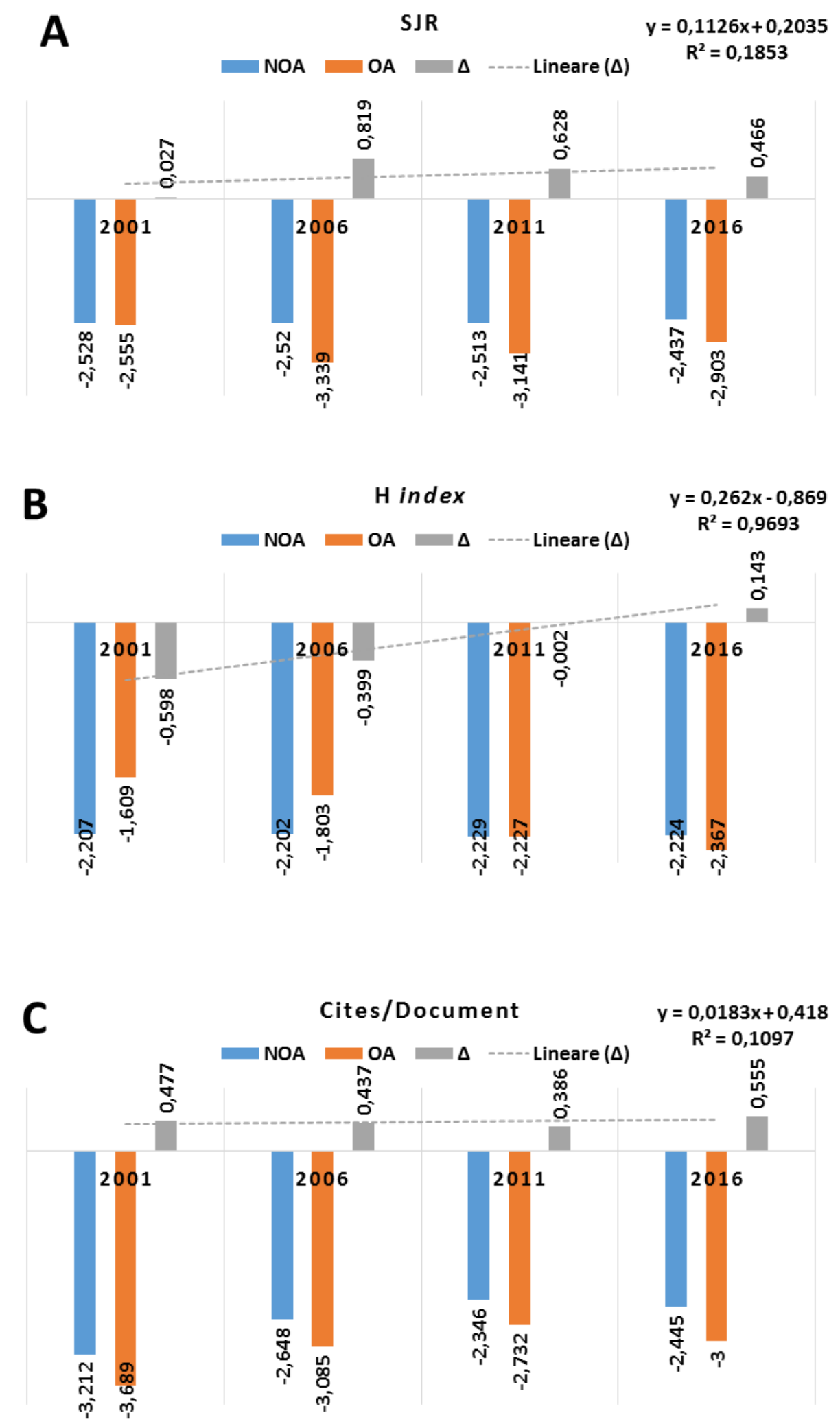

386 Figure 6: graphs showing the exonents of bibliometric parametrs (SJR, H index, and Cites/Document) in 2001, 2006, 